\title{
Outcomes After Failed Pneumatic Retinopexy for Retinal Detachment
}

\section{Citation}

Anaya, Joseph Anthony. 2017. Outcomes After Failed Pneumatic Retinopexy for Retinal Detachment. Doctoral dissertation, Harvard Medical School.

\section{Permanent link}

http://nrs.harvard.edu/urn-3:HUL.InstRepos:40621401

\section{Terms of Use}

This article was downloaded from Harvard University's DASH repository, and is made available under the terms and conditions applicable to Other Posted Material, as set forth at http:// nrs.harvard.edu/urn-3:HUL.InstRepos:dash.current.terms-of-use\#LAA

\section{Share Your Story}

The Harvard community has made this article openly available.

Please share how this access benefits you. Submit a story.

\section{Accessibility}




\begin{abstract}
by
Joseph Anaya

\section{Submitted in Partial Fulfillment of the Requirements for the M.D. Degree with Honors in a Special Field}

\title{
February 6, 2017
}

Area of Concentration:

Ophthalmology

Project Advisor:

Jeffrey Heier, M.D., Chirag Shah, M.D.

Author's Prior Degrees:

Bachelor of Arts

I have reviewed this thesis. It represents work done by the author under my supervision and guidance.




Objective: Provide visual and anatomic outcomes for patients with retinal detachment who failed primary pneumatic retinopexy (PR).

Design: Retrospective, single-center, consecutive case series

Subjects: Eyes with retinal detachment (RD) that failed a primary PR.

Methods: Anatomic and functional outcomes were evaluated for patients receiving treatment for failed PR. Three secondary procedures were compared including repeat PR, pars plana vitrectomy (PPV), and combined scleral buckle/pars plana vitrectomy (SB/PPV).

Main outcome measures: Anatomic reattachment and visual acuity at 1 year.

Results: Of a total of 423 primary PR's performed for RD, this study included 73 cases that failed. The overall single surgery anatomic success rate for the secondary procedure was $75 \%$; the final success rate at one year was $100 \%$. There was no statistically significant difference in success rates between repeat PR (63\%), PPV (76\%), and SB/PPV (88\%). Improvement in visual acuity was similar at one year between all three groups. Visual acuity at one year was similar between eyes undergoing PPV and SB/PPV (LogMAR VA 0.47 [20/59] for PPV and LogMAR VA $0.52[20 / 66]$ for SB/PPV, $p=0.75)$. Visual acuity at one year was better for those without macular involvement at the time of secondary procedure compared to eyes whose maculae detached (LogMAR VA 0.29 [20/39] vs LogMAR VA 0.73 [20/106], p < 0.005). Fifty percent of PR failures underwent a secondary procedure within 1 week of primary PR; $80 \%$ occurred within 1 month.

Conclusions: Anatomic success rates for secondary PR, PPV, and SB/PPV after failed PR were lower than published success rates for their use in primary RD. This suggests that a failed primary PR selects for retinal detachments that are inherently more difficult to reattach. There was a trend suggesting anatomical success rates are greater with SB/PPV than PPV and, in turn, with PPV than repeat PR. However, these differences were not statistically significant and did not translate into better visual acuity gains at one year for either procedure. A randomized 
controlled trial is necessary to best determine the most effective procedure after a failed pneumatic. 


\section{Table of Contents}

Glossary of abbreviations 5

Introduction 6

Methods 8

$\begin{array}{ll}\text { Results } & 10\end{array}$

Discussion 13

$\begin{array}{ll}\text { References } & 16\end{array}$

Tables and Figures $\quad 19$ 
Glossary of Abbreviations

PPV: pars plana vitrectomy

PR: pneumatic retinopexy

PVR: proliferative vitreoretinopathy

$\mathrm{RD}$ : retinal detachment

RPE: retinal pigment epithelium

SB/PPV: combined scleral buckle and pars plana vitrectomy

VA: visual acuity 
Introduction:

Rhegmatogenous retinal detachments (RD) occur when a tear, break, or hole enables vitreous fluid to pass underneath the neurosensory layer into the subretinal space. ${ }^{1}$ This type of retinal detachment occurs in approximately 1-2 in 10,000 individuals, with a peak incidence between the ages of $60-69 .{ }^{2}$ Common risk factors include recent onset of posterior vitreous detachment, myopia, lattice degeneration, and cataract surgery. ${ }^{2,3,4,5}$

Three surgical procedures provide the foundation for management of RD: pneumatic retinopexy (PR), scleral buckle (SB), and pars plana vitrectomy (PPV). In PR, a small gas bubble (sulfur hexafluoride or perfluoropropane) is injected into the vitreous to tamponade the retinal break, while the retinal pigment epithelial (RPE) pumps out the subretinal fluid. Cryotherapy or laser retinopexy is used to permanently seal the break. During SB, an extraocular silicone belt is encircled around the globe to externally relieve vitreoretinal traction. A segmental SB may be employed in cases where relief of vitreoretinal traction is only needed in an isolated area. SB can also include cryotherapy, laser retinopexy, external drainage and/or intraocular gas tamponade. In PPV, the surgeon uses an intraocular approach to remove the vitreous and internally relieve vitreoretinal traction, while internally reattaching the detached retina to the RPE and sealing the break(s) with cryotherapy or laser. Combined SB and PPV (SB/PPV) enables the surgeon to externally relieve vitreoretinal traction with a buckle while also manually approximating the detached retina to the RPE. Targeted laser therapy may be used in cases of small, peripheral detachments, and for retinal breaks without detachment from the RPE. ${ }^{1}$

In choosing which of these procedures is appropriate for the patient presenting with an $\mathrm{RD}$, several anatomical, outcome, complication, and logistical factors are considered. PR traditionally has been used in phakic patients with an isolated, uncomplicated, superior retinal detachment confined to a single clock-hour. ${ }^{6}$ Depending on patient selection, it has demonstrated single surgery success rates ranging from $45-90 \%$, with an average rate of about $75 \% .{ }^{7} \mathrm{~A}$ randomized, controlled trial under the traditional criteria - superior, one-clock-hour RDs without proliferative vitreoretinopathy (PVR) — demonstrated similar anatomic success rates between PR and SB and better final visual acuity with PR. ${ }^{6}$ Recently, several groups have demonstrated PR effectiveness under expanded criteria. These include pseudophakia, vitreous hemorrhage, more 
than one break separated by more than one clock-hour, and inferior detachments if special postprocedure positioning is used. ${ }^{8,9,10,11}$

Surgeons often choose SB, PPV, or combined SB/PPV for more complex retinal detachments. ${ }^{1}$ Several randomized, controlled trials and retrospective studies have investigated the effectiveness of PPV and SB. Two trials demonstrated a higher single surgery success rate for pseudophakic eyes after PPV compared to SB. ${ }^{12,13} \mathrm{~A}$ large, multicenter, international nonrandomized study conducted by the European Vitreo-Retinal Society (EVRS) further generalized the improved anatomic performance of PPV in pseudophakic eyes and of SB in phakic eyes. ${ }^{14}$ Although other randomized, controlled trials failed to replicate statistically significant differences in comparative anatomic success rates, ${ }^{15,16}$ they did demonstrate differences in functional outcomes. One demonstrated better final visual acuity in pseudophakic eyes undergoing PPV ${ }^{16}$ and one large trial demonstrated better final visual acuity in phakic eyes undergoing SB. ${ }^{15}$ Finally, a recent meta-analysis comparing the anatomic success rate of combined SB/PPV with PPV alone demonstrated a statistically significant benefit of the combined procedure. ${ }^{17}$ Although almost all studies comparing SB/PPV to PPV alone are nonrandomized and therefore vulnerable to selection bias, eyes undergoing SB/PPV demonstrated higher rates of macula-off $\mathrm{RD}$ and inferior breaks and similar frequencies of advanced PVR as compared to the PPV group. ${ }^{17,18}$

The most significant complication after PR, SB, or PPV is recurrent detachment requiring additional procedures. Additionally, in PR eyes, about 5-10\% develop PVR. ${ }^{19}$ Complications of SB include increased axial myopia, diplopia, choroidal detachment, PVR, cystoid macular edema, eyelid abnormalities, and late extrusion or infection of the buckle. ${ }^{1,6}$ Eyes undergoing $\mathrm{PPV}$, as compared to those undergoing $\mathrm{SB}$, observe more rapid cataract formation while having similar rates of PVR (15-20\%), cystoid macular edema (10-15\%), and increased postoperative intraocular pressure (8-10\%). ${ }^{1,20}$ Due to differences in patient selection, the rates of PVR in PR are not directly comparable to those observed in these studies for SB and PPV.

Logistically, PR confers a significant advantage as an office-based intervention. As SB and PPV are performed in an operating theater, obtaining access to these interventions may delay treatment and be associated with higher costs. ${ }^{10}$ Because delays in treatment may decrease visual recovery, ${ }^{21}$ and due to PR's favorable complication profile in select patients, PR is a useful procedure for patients with appropriate anatomical considerations. 
While the above studies provide insight for the management of a patient initially presenting with $\mathrm{RD}$, relatively few studies analyze outcomes in the patient cohort that fails primary PR. ${ }^{22-27}$ Ambler et al. (1990) found no statistically significant difference in the final visual outcome in the cohort of patients that fail the initial PR as compared with the group in which the initial PR succeeds. This result was confirmed by Mudvari et al. (2009). Recently, Fabian et al. (2013) found no significant difference in visual acuity outcomes between successful PR cases and those with only one additional operation, but significantly worse outcomes in the cases requiring two or more additional operations. However, their results were not stratified by procedure type. After initial PR failure, the comparative anatomic and functional outcomes for secondary PR, PPV, and combined scleral buckle and pars plana vitrectomy (SB/PPV) remains unknown. This study compares the outcomes after primary PR failure according to secondary procedure type and preoperative characteristics at the time of PR failure.

Methods:

This is a retrospective, consecutive case review conducted at a single center, the Ophthalmic Consultants of Boston (Boston, MA). Ten surgeons performed the vitreoretinal procedures. All eyes developing a recurrent or persistent retinal detachment after primary PR from July 1, 2009 to July 1, 2014, were included in the study. A primary PR involved cryo therapy to the break(s) followed by an intraocular gas injection. A failed PR was defined as one where the eye required a repeat PR, or PPV or SB/PPV for persistent or recurrent retinal detachment within 1 year of the primary PR. Only one eye meeting inclusion criteria underwent scleral buckling without PPV after failed PR, and thus this case was excluded in analysis. Eyes requiring only additional laser or cryo therapy in the immediate post-op period were not considered pneumatic failures. Patients were followed to one year. Five patients had less than one year but more than six months follow-up; therefore, the last observation for these patients was carried forward to one year. Those who did not require an additional PR or vitreoretinal procedure for retinal detachment within one year were excluded.

Cases occurring from July 1, 2009 to May 1, 2012 were included in a prior published study that analyzed success rates and preoperative characteristics of the primary pneumatic 
procedure. ${ }^{10}$ The current study adds patients through July 1, 2014, and is unique in that it evaluates outcomes specifically in patients who fail primary pneumatic retinopexy.

Demographic variables collected included age, gender, right or left eye, and history of prior laser or cryo retinal therapy. Variables collected for the presentations at initial PR and time of PR failure were macula status, lens status, presence of posterior vitreous detachment, presence of lattice degeneration, presence of vitreous hemorrhage, presence of bridging vessel, whether there was visible traction on the tear, the presence of proliferative vitreoretinopathy, and the performance of additional procedures. The reason for failure was deduced at the time of PR failure according to the surgeon's retinal exam and assessment. Reasons for failure included 1) missed or new break, 2) persistent detachment, or 3) proliferative vitreoretinopathy. Visual acuity (VA) was collected at primary presentation, time of PR failure, and one-year follow-up available to July 1, 2015. VA was recorded on a Snellen chart as the better-observed vision between spectacle-corrected and pin-hole measures. Development of PVR and date of cataract surgery were also collected longitudinally for each patient.

Descriptive statistics were used to summarize the demographic features, preoperative characteristics, visual presentations, and functional and anatomic outcomes. Composite analysis on all surgeries and subgroup analysis was performed for cases undergoing pneumatic retinopexy, pars plana vitrectomy, and combined scleral buckle/pars plana vitrectomy. Functional outcomes were also subanalyzed according to macula status for all procedures and lens status for PPV procedures. The latter subanalysis was an attempt to control for cataract development among phakic patients. Anatomic success rates were compared overall and on a procedure-specific basis for all patients, phakic patients, pseudophakic patients, macula-on patients at the time of PR failure, and macula-off patients at the time of PR failure.

Statistical analyses were performed using Stata 12 (College Station, TX) and Microsoft Excel (Redmond, WA). A 2-tailed test of proportion was used to compare rates and other discrete outcomes. A 2-tailed paired Student t-test was used to compare visual acuity presentation differences and outcomes.

IRB approval was obtained from Chesapeake Institutional Review Board (Columbia, MD). 
Results:

\section{Demographics}

Of 426 PR cases that occurred within the study period, 73 cases met inclusion and exclusion criteria. Previous publication showed that the initial PR success rate for this group of surgeons is $79 \%{ }^{5}$ Demographics of the included cases are displayed in Table 1 (available at http://aaojournal.org) and are stratified by secondary procedure.

Pre-operative characteristics at primary presentations and time of PR failure are presented in Table 2 for each type of secondary procedure. Overall, $77 \%$ and $66 \%$ of maculae were attached at the primary presentation and time of PR failure, respectively. A similar number of eyes undergoing secondary PPV or SB/PPV had attached maculae $(61 \%$ vs $63 \%, \mathrm{p}=1)$, but a non-significantly higher percentage of eyes undergoing repeat PR had attached maculae $(81 \%, p$ $=0.23$ ). A higher percentage of eyes undergoing SB/PPV had PVR compared to eyes undergoing PPV, with borderline statistical significance (19\% for SB/PPV vs $2 \%$ for PPV, $p=0.06$; $19 \%$ for $\mathrm{SB} / \mathrm{PPV}$ vs $0 \%$ for repeat PR, $\mathrm{p}=0.22$ ).

\section{Time to failure of initial PR:}

The cumulative $50^{\text {th }}$ percentile for time to failure of the initial PR was 6 days and ranged from 1 to 250 days (Figure 1). The majority of PR failures $(80 \%)$ occurred within one month of primary PR. Twelve eyes $(21 \%)$ with attached maculae at the time of primary PR had a detached macula at the time of their secondary procedure. The cumulative $50^{\text {th }}$ percentile to macula detachment was 7 days and ranged from 1 to 157 days (Figure 1).

\section{Anatomic Outcomes:}

No significant differences were observed in single surgery anatomic success rates for the secondary procedure when stratified by procedure type (75\% overall; $63 \%$ repeat PR vs $76 \%$ PPV, $p=0.34$; repeat PR vs. 88\% SB/PPV, $p=0.22$; PPV vs. SB/PPV, $p=0.48$, Figure 2). There were no differences in anatomic success rates between phakic versus pseudophakic eyes $(79 \%$ vs. $68 \%, p=0.39)$, nor for macula-attached versus macula-detached eyes $(79 \%$ vs. $65 \%, p$ $=0.25)$.

In total, 100 surgeries after the initial failed PR were performed on the 73 eyes ( 7 additional procedures for the 16 eyes in the repeat PR group vs 3 additional procedures for the 
16 eyes in the SB/PPV group, $\mathrm{p}=0.24 ; 17$ additional procedures for the 41 eyes in the PPV group vs 3 additional procedures for the $16 \mathrm{SB} / \mathrm{PPV}$ eyes, $\mathrm{p}=0.24$ ). One PPV eyes received a total four procedures and one received a total of six procedures. Final anatomic success at one year was $100 \%$.

\section{Functional Outcomes:}

Visual acuities at initial presentation, at the time of PR failure, and at one-year follow-up are displayed in Table 3. The overall mean visual acuity one-year after surgery for failed PR improved, with statistical significance, from the visual acuity at the time of PR failure (1 year follow-up LogMAR 0.43 [20/54] vs time of PR failure LogMar VA 0.60 [20/80], p=0.03). However, the final visual acuity did not improve compared to the visual acuity at initial presentation (initial presentation LogMar VA 0.52 [20/66] vs 1 year follow-up LogMAR 0.43 [20/54], $p=0.28)$. When stratified by type of secondary procedure, the final visual acuity after $\mathrm{PPV}$ or SB/PPV remained similar to that at initial presentation, as well as at the time of PR failure (PPV eyes one year follow-up LogMAR 0.47 [20/59] vs initial presentation LogMAR 0.50 [20/63], $\mathrm{p}=0.80$, vs time of PR failure logMAR 0.67 [20/94], $\mathrm{p}=0.10 ; \mathrm{SB} / \mathrm{PPV}$ one year follow-up LogMAR 0.52 [20/66] vs initial presentation LogMAR 0.50 [20/63], $\mathrm{p}=0.88$, vs time of PR failure LogMAR 0.69 [20/98], $\mathrm{p}=0.50$ ).

Eyes undergoing repeat PR had better vision at time of PR failure and at one-year followup compared to those undergoing PPV or SB/PPV (Repeat PR LogMAR VA 0.32 [20/42] vs PPV and SB/PPV combined LogMAR VA 0.68 [20/94], $p=0.02$ ). This finding suggests a selection bias favoring repeat PR for less extensive RD's failing initial PR. Each procedure group experienced similar non-significant improvements in visual acuity from time of PR failure to one-year follow-up (Table 3).

When stratifying by three categories of visual acuity, 20/20 to 20/40,20/50 to 20/100, and 20/200 and worse, there were no differences in distribution at initial presentation, at time of PR failure, and one-year follow-up (Figure 3). The proportion of patients with 20/20 to 20/40 vision for each respective procedure group was similar at the time of PR failure and at one-year follow-up ( $79 \%$ vs $81 \%$ for repeat PR, $p=1 ; 46 \%$ vs $63 \%$ for PPV, $p=0.18 ; 50 \%$ vs $64 \%$ for $\mathrm{SB} / \mathrm{PPV}, \mathrm{p}=1)$. These were not necessarily the same eyes, however. The percent of eyes with visual improvement between PR failure and one-year was similar across groups (50\% for PR, 
$51 \%$ for PPV, and $50 \%$ for SB/PPV) and some eyes maintained the same vision $(25 \%$ in $\mathrm{PR}$, $22 \%$ in $\mathrm{PPV}$, and $7 \%$ in $\mathrm{SB} / \mathrm{PPV})$.

\section{Macula Status}

Eyes with detached maculae at the time of PR failure had worse vision compared to eyes with attached maculae (maculae-detached LogMAR VA 1.11 [20/256] vs maculae-attached LogMAR VA 0.37 [20/47], p <0.0005). At one year follow-up, these maculae-detached eyes had substantially worse vision compared to eyes with attached maculae (maculae-detached LogMAR VA 0.73 [20/106] vs maculae-attached LogMAR VA 0.29 [20/39], p <0.005). Twelve of 56 eyes $(21 \%)$ with attached maculae at initial presentation had detached maculae at time of failure PR. Compared to those whose maculae remained attached, eyes developing detached maculae had worse vision at time of PR failure, (eyes developing detached maculae LogMAR VA 1.10 [20/250] vs maculae remaining attached LogMAR VA 0.37 [20/47], p < 0.005), and a trend toward worse vision at one-year follow-up (eyes developing detached maculae LogMAR VA 0.59 [20/78] vs maculae remaining attached LogMAR VA 0.29 [20/39], $p=0.14$ ).

\section{Lens status and cataract development}

Similar visual outcomes were observed for PPV patients regardless of lens status at the time of their secondary procedure. Phakic and pseudophakic patients had similar visual acuity at time of PR failure (phakic LogMAR VA 0.61 [20/81] vs pseudophakic LogMAR VA 0.76 [20/114], p = 0.65) and one-year follow-up (phakic LogMAR VA 0.45 [20/56] vs pseudophakic LogMAR VA 0.52 [20/66], p = 0.68). Additionally, patients who were phakic at time of PR failure and remained phakic at one-year follow-up $(n=9)$ compared to those who were phakic at time of PR failure and pseudophakic at one-year follow-up $(n=15)$ observed similar visual acuity at time of PR failure (eyes remaining phakic LogMAR VA 0.61 [20/81] vs eyes receiving cataract extraction LogMAR VA 0.62 [20/83], p =0.96), and one-year follow-up (eyes remaining phakic LogMAR VA 0.38 [20/48] vs eyes receiving cataract extraction LogMAR VA 0.40 [20/50], $\mathrm{p}=0.93$ ). Each group had similar improvements in VA at one-year compared to time of PR failure (logMAR VA -0.22 vs $-0.22, \mathrm{p}=0.99)$.

Among patients who were phakic at time of PR failure (10 repeat PR eyes, 26 PPV eyes, and $15 \mathrm{SB} / \mathrm{PPV}$ eyes), cataract extraction occurred at a statistically higher rate in the PPV eyes 
compared to the repeat PR eyes ( $62 \%$ vs $10 \%, \mathrm{p}=0.008)$. The cataract extraction rate for $\mathrm{SB} / \mathrm{PPV}$ eyes compared to PR eyes trended toward statistical significance ( $47 \%$ vs $10 \%, \mathrm{p}=$ 0.09). Statistically comparable rates of cataract extraction were observed for the PPV eyes compared to the SB/PPV eyes $(62 \%$, vs $47 \%, \mathrm{p}=0.52)$

\section{Discussion}

Approximately one quarter of eyes undergoing PR for retinal detachment will fail. ${ }^{7}$ The overall single surgery success rate for the secondary procedure, $75 \%$, is similar to the initial PR, and is consistent with prior reports. ${ }^{10,26}$ After failed PR, the success rates of repeat PR (63\%), PPV (76\%), and SB/PPV (88\%) are generally lower than published reports of the individual procedures for primary RD ( $75 \%$ for PR and greater than $90 \%$ for PPV or SB/PPV). ${ }^{7,14,28,29}$ These findings suggest that failure of primary PR selects for RD's that may be more difficult to reattach. Indeed, the 73 eyes failing PR in this series undergoing a secondary procedure required an additional 27 procedures for retinal reattachment, with two eyes requiring four or more procedures.

Functionally, eyes failing PR had no change in visual acuity at 1 year after secondary $\mathrm{PPV}$ or SB/PPV, contrary to the improvement in visual acuity noted after successful PR. ${ }^{10}$ It appears a failed PR may portend a worse visual outcome compared to those that succeed. Among 107 successfully reattached RD's after PR, the visual acuity at 6 months was LogMAR 0.20 [20/32] (unpublished data). ${ }^{10}$ In the present study, the visual acuity 1 year after a failed PR for eyes undergoing PPV or SB/PPV (LogMAR VA 0.49 [20/61]) was statistically worse than visual acuity 6 months after successful PR $(p=0.0002)$. Nonetheless, while it is difficult to make comparisons across studies with disparate methods, this visual result is comparable to functional results achieved when PPV and SB/PPV are employed as the initial procedure (LogMAR VA 0.4 $[20 / 50]-0.5[20 / 63]) .{ }^{13,28,30}$

Though there was no statistical difference in the success rates for secondary RD based on type of surgery, there was a trend toward higher success rates with more extensive surgery with $88 \%$ of eyes undergoing SB/PPV remaining attached without further surgery. Moreover, failed PR cases with PVR were overrepresented in the SB/PPV group with borderline statistical significance. This may suggest a selection bias that could possibly lower the anatomic and 
functional success of SB/PPV. But the above results suggest that SB/PPV, though more invasive than PPV alone or repeat PR, may be worth considering after failed PR, particularly in cases of PVR or for those with greater clinical concern. It is important to realize, however, that there was no difference in visual acuity at 1 year between eyes undergoing SB/PPV and PPV alone. It remains unclear which procedure should be performed in the event of a failed PR. A randomized prospective study would help determine the true difference in success rates for a repeat PR, PPV, $\mathrm{SB}$, or a SB/PPV after failed PR.

Repeating PR after failure of the primary PR can be a useful option in select cases, albeit with lower success rates than more extensive surgery. In the present series, eyes undergoing repeat PR had better visual acuity than eyes undergoing PPV or SB/PPV, were more likely to have attached maculae, and had no PVR. Thus there appeared to be a bias of offering repeat PR to “milder" recurrent RD's. Nonetheless, repeat PR remains a useful tool for such milder cases given that the $63 \%$ of success rate in the present study is not statistically different than the $75 \%$ literature success rate for initial PR, and that eyes undergoing repeat PR experienced the same visual acuity improvements as more extensive surgery.

Interestingly, $21 \%$ of macula-attached patients who failed PR, or about $4 \%$ of all maculaattached patients who receive PR for primary retinal detachment, will present with macular involvement at the time of retinal redetachment. Moreover, about half of patients who fail PR do so within the first week. Thus, it may be worthwhile to follow macula-attached patients undergoing PR closely within the first week to possibly intervene in cases that are failing before the macula is involved.

There are several limitations to the current study. Its retrospective nature makes it vulnerable to the common potential pitfalls of such studies, such as surgeon preference and selection bias, as demonstrated by the observation that more favorable eyes tended to receive repeat PR and a greater number of eyes with PVR received SB/PPV. The three groups, repeat PR, PPV, SB/PPV, did not have a balanced number of patients, and there was only one patient who received SB alone after failed PR. Duration of macular detachment prior to presentation was not well-documented in the medical record and could not reliably be assessed. In addition, this study was not able to determine best-corrected visual acuity, or control for other variables that may limit visual acuity, such as cataract, corneal pathology or macular pathology. However, the subanalysis of PPV patients stratified by lens status showed that the results did not appreciably 
change for phakic or pseudophakic at one-year follow-up. Finally, it is possible that eyes in the present study would have experienced additional visual improvement if they were followed longer than one year, as suggested by the randomized, controlled trial comparing PR with SB, which found a 10\% improvement in vision between 6 and 24 months in both groups. ${ }^{31}$

In conclusion, retinal detachments failing PR in this series required more than two total procedures $25 \%$ of the time. Functionally, the visual acuity at one year is similar to that at initial presentation. Though there was no statistical difference between repeat PR, PPV, and SB/PPV success rates after PR failure, there is a trend toward higher success rates with more extensive surgery, albeit without difference in final visual acuity between PPV and SB/PPV. A randomized controlled trial is necessary to best determine the most effective procedure after a failed pneumatic.

\section{Acknowledgements:}

I extend my greatest gratitude to my mentors Chirag Shah, Michael Morley, and Jeffrey Heier, who provided invaluable guidance throughout this project and who afforded me an incredible start to a fulfilling career in ophthalmology. Financial support for the IRB application was provided by the Center for Eye Research and Education. 


\section{References:}

1. D'Amico DJ. Primary retinal detachment. New England Journal of Medicine 2008; 359: 2346-54.

2. Rowe JA, Erie JC, Baratz KH, Hodge DO, Gray DT, Butterfield L, Robertson DM. Retinal detachment in Olmsted County, Minnesota, 1976-1995. Ophthalmology 1999; 106: 154159.

3. Byer NE. Long-term natural history of lattice degeneration of the retina. Ophthalmology 1989; 96: 1396-1402.

4. Hikichi T, Trempe CL, Schepens CL. Posterior vitreous detachment as a risk factor for retinal detachment. Ophthalmology 1995; 102: 527.

5. Byer NE. Natural history of posterior vitreous detachment with early management as the premier line of defense against retinal detachment. Ophthalmology 1994; 101: 1503.

6. Tornambe PE, Hilton GF, The Retinal Detachment Study Group. A multicenter randomized controlled clinical trial comparing pneumatic retinopexy with scleral buckling. Ophthalmology 1989; 96: 772-784.

7. Chan CK, Lin SG, Nuthi AS, Salib DM. Pneumatic retinopexy for the repair of retinal detachments: a comprehensive review (1986-2007). Surv Ophthalmol 2008;53: 443-78.

8. Schaal S, Sherman MP, Barr CC, Kaplan HJ. Primary retinal detachment repair: comparison of 1-year outcomes of four surgical techniques. Retina 2011;31:1500-4.

9. Davis MJ, Mudvari SS, Shott S, Rezaei KA. Clinical characteristics affecting the outcome of pneumatic retinopexy. Arch Ophthalmol 2011;129:163-6.

10. Goldman DR, Shah CP, Heier JS. Expanded Criteria for Pneumatic Retinopexy and Potential Cost Savings. Ophthalmology 2014; 121: 318-326.

11. Hwang JF, Chen SN, Lin CJ. Treatment of inferior rhegmatogenous retinal detachment by pneumatic retinopexy technique. Retina 2011;31:257-61.

12. Brazitikos PD, Androudi S, Christen WG, Stangos NT. Primary pars plana vitrectomy versus scleral buckle surgery for the treatment of pseudophakic retinal detachment. Retina 2005; 25: 957-964.

13. Heiman H, Bartz-Schmidt UL, Bornfeld N, et al. for the Scleral Buckling versus Primary Vitrectomy in Rhegmatogenous Retinal Detachment Study Group. Scleral Buckling versus 
Primary Vitrectomy in Rhegmatogenous Retinal Detachment: a prospective randomized multicenter clinical study. Ophthalmology 2007 Dec; 114 (12): 2142-54.

14. Adelman RA, Parnes AJ, Sipperley JO, Ducournau D, for the European Vitreo-Retinal Society (EVRS) Retinal Detachment Study Group. Strategy for the Management of Complex Retinal Detachments. Ophthalmology 2013; 120: 1809-1813.

15. Ahmadieh H, Moradian S, Faghihi H, Parvaresh MM, Ghanbari H, Mehryar M, Heidari E, Behboudi, Banaee T, Golestan, for the Pseudophakic and Aphakic Retinal Detachment (PARD) Study Group. Anatomic and visual outcomes of scleral buckling versus primary vitrectomy in pseudophakic and aphakic retinal detachment. Ophthalmology 2005; 112: 1421-1429.

16. Sharma YR, Karunanithi S, Azad RV et al. Functional and anatomic outcome of scleral buckling versus primary vitrectomy in pseudophakic retinal detachment. Acta Ophthalmol Scand 2005; 83: 293-297.

17. Totsuka K, Inui H, Roggie MF, Hirasawa K, Noda Y, Ueta T. Supplemental scleral buckle in vitrectomy for the repair of rhegmatogeous retinal detachment,; A Systematic Review of Literature and Meta-Analysis. Retina 2015; 35: 2423-2431.

18. Adelman, RA, Parnes AJ, Michalewska Z, Ducournau D, for the European Vitreo-Retinal Society (EVRS) Retinal Detachment Study Group. Clinical Variable Associated with Failure of Retinal Detachment Repair. Ophthalmology 2014; 121: 1715-1719.

19. Hilton GF, Tornambe PE, The Retinal Detachment Study Group. Pneumatic retinopexy and analysis of intraoperative and postoperative complications. Retina 1991; 11: 285-294.

20. Soni C, Hainsworth DP, Almony A. Surgical management of rhegmatogenous retinal detachment: a meta-analysis of randomized controlled trials. Ophthalmology 2013; 120:1440-1447.

21. Diederen RM, La Heij EC, Kessels AGH, Goezinne F, Liem ATA, Hendrikse F. Scleral buckling surgery after macula-off retinal detachment: worse visual outcome after more than 6 days. Ophthalmology 2007; 114: 705-709.

22. Grizzard WS, Hilton GF, Hammer ME, Taren D, Brinton DA. Pneumatic retinopexy failures. Ophthalmology 1995; 102: 929-936. 
23. Gilca M, Duval R, Goodyear E, et al. Factors associated with outcomes of pneumatic retinopexy for rhegmatogenous retinal detachments: a retrospective review of 422 cases. Retina 2014 Apr; 34(4): 393-9.

24. Modi YS, Epstein A, Flynn HW Jr., et al. Outcomes and complications of Pneumatic Retinopexy Over a 12-Year Period. Opthalmic Surg Lasers Imaging Retina 2014; 45: 132137.

25. Fabian ID, Kinori M, Efrati M, et al. Pneumatic Retinopexy for the Repair of Primary Rhegmatogenous Retinal Detachment. JAMA Ophthalmol 2013. 131(2): 166-171.

26. Mudvari SS, Ravage ZB, Rezaei KA. Retinal Detachment After Primary Pneumatic Retinopexy. Retina 29: 1474-1478, 2009.

27. Ambler JS, Meyers SM, Zegarra H, Paranandi L. Reoperations and visual results after failed pneumatic retinopexy. Ophthalmology 1990; 97:786-790.

28. Weichel ED, Martidis A, Fineman MS, et al. Pars plana vitrectomy versus combined pars plana vitrectomy-scleral buckle for primary repair of pseudophakic retinal detachment. Ophthalmology 2006;113:2033-40.

29. Stangos AN, Petropoulos IK, Brozou CG, et al. Pars-plana vitrectomy alone vs vitrectomy with scleral buckling for primary rhegmatogenous pseudophakic retinal detachment. Am J Ophthalmol 2004;138:952-8.

30. Kinori M, Moisseiev E, Shoshany N, Fabian ID, Skaat A, Barak A, Loewenstein A, Moisseiev J._Comparison of pars plana vitrectomy with and without scleral buckle for the repair of primary rhegmatogenousretinal detachment. Am J Ophthalmol. 2011 Aug;152(2):291-297.

31. Tornambe PE, Hilton GF, Brinton DA, et al. A Two-year follow-up study of the multicenter clinical trial comparing pneumatic retinopexy with scleral buckling. Ophthalmology 1991; 98: 1115-1123. 
Tables and Figures:

Table 1. Demographics

\begin{tabular}{|l|c|c|c|c|}
\hline $\begin{array}{c}\text { Demographic } \\
\text { Features }\end{array}$ & $\begin{array}{c}\text { Overall } \\
\mathrm{n}\end{array}$ & $\begin{array}{c}\text { Repeat PR } \\
\mathrm{n}\end{array}$ & $\begin{array}{c}\text { PPV } \\
\mathrm{n}\end{array}$ & $\begin{array}{c}\text { SB/PPV } \\
\mathrm{n}\end{array}$ \\
\hline Number of eyes & 73 & 16 & 41 & 16 \\
\hline Mean age (y) & 59 & 55 & 61 & 57 \\
\hline Male & 52 & 15 & 26 & 11 \\
\hline Right Eye & 32 & 8 & 15 & 4 \\
\hline Prior LRP & 13 & 3 & 6 & 1 \\
\hline Prior cryo & 4 & 0 & 3 & \\
\hline
\end{tabular}


Table 2. Pre-operative characteristics of eyes at time of pneumatic retinopexy failure

\begin{tabular}{|l|c|c|c|c|}
\hline & All & $\begin{array}{c}\text { Repeat } \\
\text { PR }\end{array}$ & PPV & SB/PPV \\
\hline Macula status & & & & \\
\hline Attached & $48(66)$ & $13(81)$ & $25(61)$ & $10(63)$ \\
\hline Detached & $25(34)$ & $3(19)$ & $16(39)$ & $6(38)$ \\
\hline Lens Status & & & & \\
\hline Phakic & $48(66)$ & $9(56)$ & $25(60)$ & $14(88)$ \\
\hline Pseudophakic & $25(34)$ & $7(44)$ & $16(40)$ & $2(13)$ \\
\hline Posterior vitreous detachment & $46(63)$ & $7(44)$ & $30(73)$ & $9(56)$ \\
\hline Any lattice degeneration & $12(16)$ & $3(31)$ & $5(12)$ & $4(25)$ \\
\hline Proliferative vitreoretinopathy & $4(5)$ & $0(0)$ & $1(2)$ & $3(19)$ \\
\hline Reason for PR failure & & & & \\
\hline Missed or new break & $27(37)$ & $8(50)$ & $14(30)$ & $5(31)$ \\
Persistent detachment & $42(58)$ & $8(50)$ & $26(60)$ & $8(50)$ \\
PVR & $4(5)$ & $0(0)$ & $1(2)$ & $3(19)$ \\
\hline
\end{tabular}

$\mathrm{n}(\%)$ where $\%$ of "All" cases is the percent of total cases. $\%$ for specific procedures is the $\%$ of cases for that procedure. $\mathrm{PR}=$ pneumatic retinopexy, PPV = pars plana vitrectomy, SB/PPV = combined scleral buckle/pars plana vitrectomy. 


\begin{tabular}{|c|c|c|c|c|c|}
\hline & $\begin{array}{c}\text { Initial } \\
\text { Presentation } \\
\text { LogMAR VA } \\
\text { Mean (Snellen) }\end{array}$ & $\begin{array}{l}\text { Time of PR } \\
\text { failure } \\
\text { LogMAR VA } \\
\text { Mean (Snellen) }\end{array}$ & $\begin{array}{c}1 \text { year follow-up } \\
\text { LogMAR VA } \\
\text { Mean (Snellen) }\end{array}$ & $\begin{array}{c}\text { p-value } \\
\text { comparing } \\
\text { VA } 1 \text { year } \\
\text { follow-up to } \\
\text { initial } \\
\text { presentation }\end{array}$ & $\begin{array}{c}\text { p-value } \\
\text { comparing VA } \\
\text { at } 1 \text { year } \\
\text { follow-up to } \\
\text { time of PR } \\
\text { failure }\end{array}$ \\
\hline All eyes $(n=73)$ & $0.52(20 / 66)$ & $0.60(20 / 80)$ & $0.43(20 / 54)$ & 0.28 & 0.03 \\
\hline Repeat PR $(n=16)$ & $0.59(20 / 77)$ & $0.32(20 / 42)$ & $0.22(20 / 33)$ & 0.04 & 0.11 \\
\hline $\operatorname{PPV}(n=41)$ & $0.50(20 / 63)$ & $0.67(20 / 94)$ & $0.47(20 / 59)$ & 0.80 & 0.10 \\
\hline $\mathrm{SB} / \mathrm{PPV}(\mathrm{n}=16)$ & $0.50(20 / 63)$ & $0.69(20 / 98)$ & $0.52(20 / 66)$ & 0.88 & 0.50 \\
\hline Macula-detached, all procedures $(\mathrm{n}=48)$ & $0.94(20 / 173)$ & $1.11(20 / 256)^{*}$ & $0.73(20 / 106)^{\dagger}$ & 0.28 & 0.04 \\
\hline Macula-attached, all procedures $(n=25)$ & $0.33(20 / 42)$ & $0.37(20 / 47)^{* \#}$ & $0.29(20 / 39)^{\dagger \dagger}$ & 0.69 & 0.34 \\
\hline $\begin{array}{l}\text { Macula attached at initial presentation, detached at } \\
\text { time of PR failure }(\mathrm{n}=12)\end{array}$ & $0.36(20 / 46)$ & $1.10(20 / 250)^{\#}$ & $0.59(20 / 78)^{+}$ & 0.39 & 0.08 \\
\hline Pseudophakic at time of PR failure, PPV $(n=16)$ & $0.56(20 / 73)$ & $0.76(20 / 114)$ & $0.52(20 / 66)$ & 0.54 & 0.22 \\
\hline Phakic at time of PR failure, PPV $(n=25)$ & $0.46(20 / 58)$ & $0.61(20 / 81)$ & $0.45(20 / 56)$ & 0.84 & 0.26 \\
\hline
\end{tabular}

Table 3. Presentations and Functional Outcomes in Eyes Failing Primary Pneumatic Retinopexy.

${ }^{*} \mathrm{p}<0.00005$, comparing VA at time of PR failure for eyes that are maculae detached vs attached at time of PR failure.

${ }^{\dagger} \mathrm{p}<0.005$, comparing one year follow-up VA for eyes that are maculae detached vs attached at time of PR failure.

${ }^{\#} \mathrm{p}<0.005$, comparing VA at time of PR failure for maculae attached eyes compared to eyes developing detached maculae.

$t_{p}=0.14$, comparing one-year follow-up VA for maculae attached eyes to eyes developing detached maculae.

$\mathrm{PR}=$ pneumatic retinopexy, $\mathrm{PPV}=$ pars plana vitrectomy, $\mathrm{SB} / \mathrm{PPV}=$ combined scleral buckle/pars plana vitrectomy, $\mathrm{VA}=\mathrm{visual}$ acuity. 


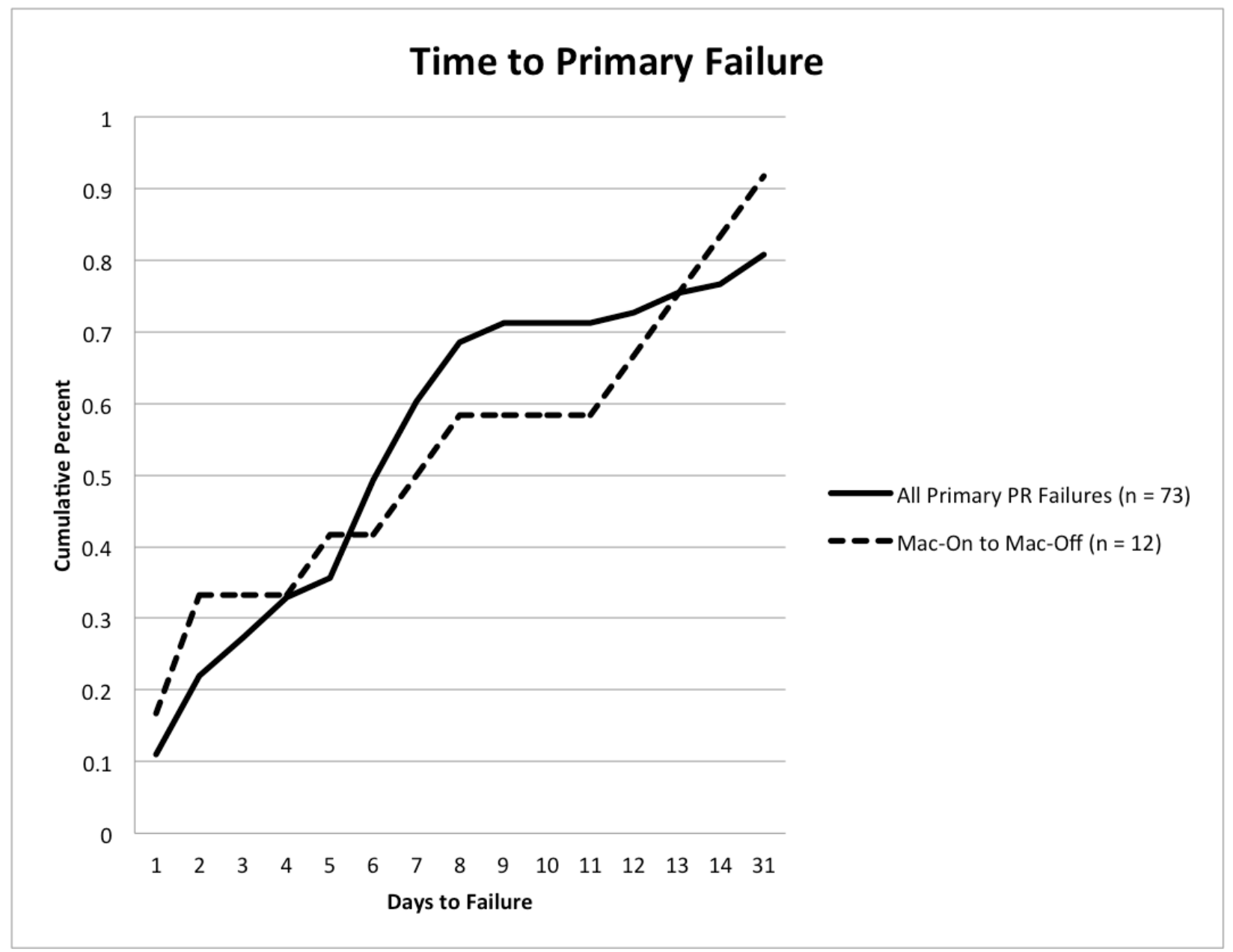

Figure 1. Time to Primary Failure. The solid line shows the cumulative percentage of eyes failing primary pneumatic retinopexy $(\mathrm{PR})$ over time $(\mathrm{n}=73)$. Of primary PR failures, approximately $50 \%$ failed by day 6 and $80 \%$ failed by day 31 . The dashed line shows the cumulative percentage of eyes failing primary PR that were maculae-attached at the time of initial PR and developed detached maculae by the time of failure $(n=12)$. Of these eyes, $50 \%$ fail by day 7 and approximately $90 \%$ fail by day 31 . 


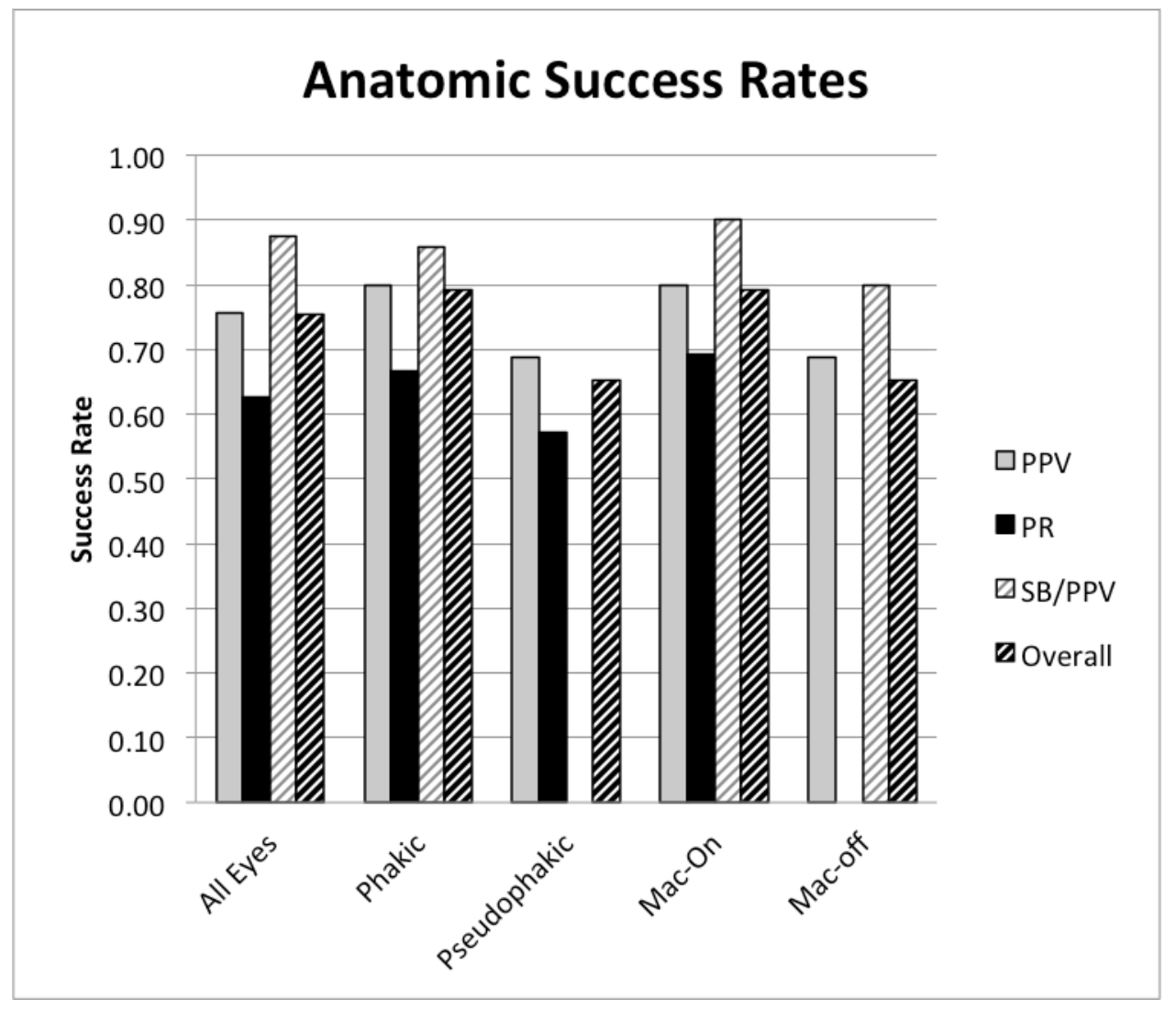

Figure 2. Anatomic outcomes by procedure and pre-operative characteristics. Each bar shows the anatomic success rate according to procedure and pre-operative characteristics. For eyes undergoing pars plana vitrectomy (PPV), the gray bar shows success rates of $76 \%$ for all eyes (n $=41), 80 \%$ for phakic eyes $(\mathrm{n}=25), 69 \%$ for pseudophakic eyes $(\mathrm{n}=16), 80 \%$ for maculaattached eyes $(n=25)$, and $69 \%$ for macula-detached eyes $(n=16)$. For eyes undergoing repeat pneumatic retinopexy (PR), the black bar shows success rates of $63 \%$ for all eyes $(n=16), 67 \%$ for phakic eyes $(n=9), 57 \%$ for pseudophakic eyes $(n=7)$, and $69 \%$ for macula-attached eyes $(n$ =13). All 3 macula-detached eyes that underwent repeat PR failed and are not shown. For eyes undergoing combined scleral buckle/pars plana vitrectomy (SB/PPV), the gray-striped bar shows success rates of $88 \%$ for all eyes $(n=16), 86 \%$ for phakic eyes $(n=14), 90 \%$ for maculaattached eyes $(n=10)$, and $80 \%$ for macula-detached eyes $(n=6) .2$ SB/PPV pseudophakic patients succeeded and are not shown. Taking a weighted average of all procedures, the overall success rate was $75 \%$ for all eyes, $79 \%$ for phakic eyes $(n=48), 65 \%$ for pseudophakic eyes $(n=$ $25), 79 \%$ for macula-attached eyes $(n=28)$, and $65 \%$ for macula-detached eyes $(n=25)$. There were no statistically significant differences between these rates. 


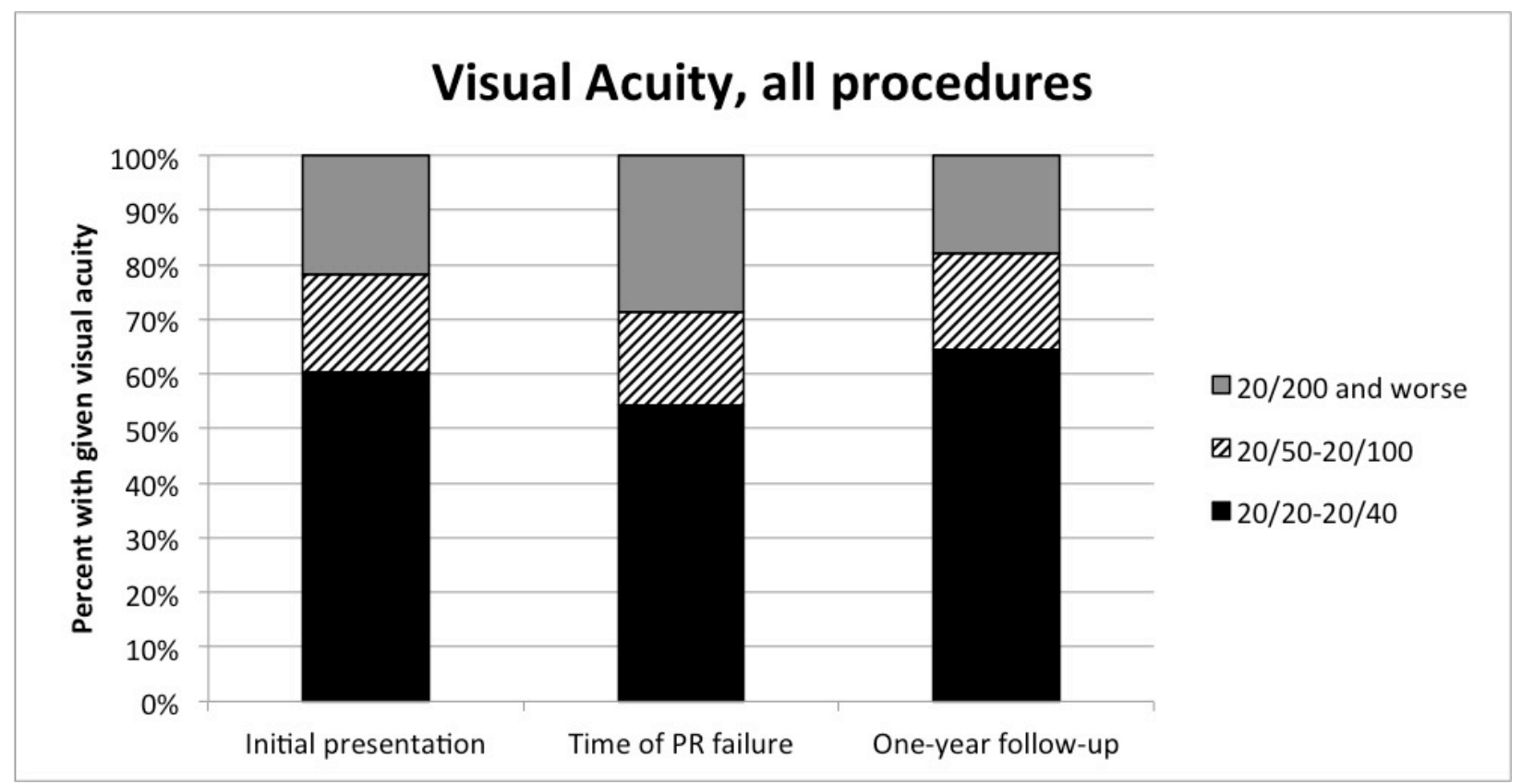

Figure 3. Distribution of Visual Acuity. Each bar shows the distribution of visual acuity at initial presentation, time of primary pneumatic retinopexy (PR) failure, and one-year follow-up. The black filling shows the percentage of patients that demonstrated $20 / 20$ to $20 / 40$ vision (60\% at initial presentation, 54\% at time of PR failure, and 64\% at one-year follow-up). The blackstriped filling shows the percentage of patients that demonstrated 20/50 to 20/100 vision (18\% at initial presentation, $17 \%$ at time of PR failure, $18 \%$ at one-year follow-up). The gray filling shows the percentage of patients that demonstrated $20 / 200$ and worse vision $(22 \%$ at initial presentation, $29 \%$ at time of PR failure, and 18\% at one-year follow-up). 